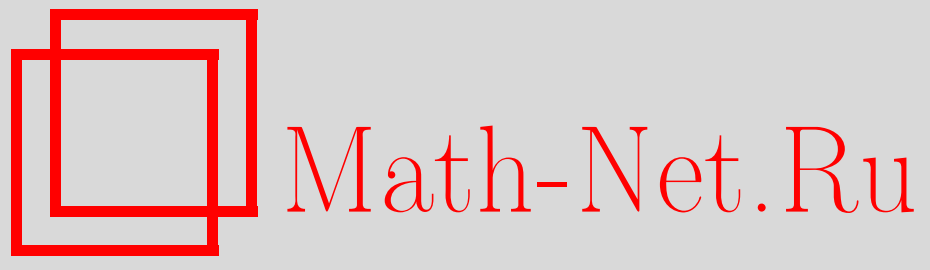

С. Г. Бобков, Некоторые обобщения результатов Ю. В. Прохорова о неравенствах типа Хинчина для полиномов, Теория вероятн. и ее примен., 2000, том 45, выпуск 4, 745-748

DOI: https://doi.org/10.4213/tvp503

Использование Общероссийского математического портала Math-Net.Ru подразумевает, что вы прочитали и согласны с пользовательским соглашением http://www . mathnet.ru/rus/agreement

Параметры загрузки:

IP : 54.224 .187 .69

26 апреля 2023 г., $12: 44: 30$

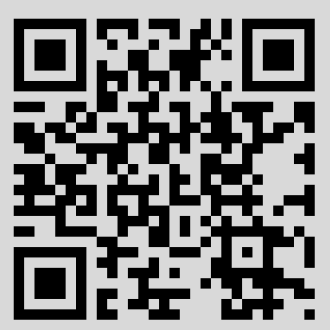


(C) 2000 r. БОБКОВ C. . $^{*}$

\title{
НЕКОТОРЫЕ ОБОБЩЕНИЯ РЕЗУЛЬТАТОВ Ю. В. ПРОХОРОВА $О$ НЕРАВЕНСТВАХ ТИПА ХИНЧИНА ДЛЯ ПОЛИНОМОВ ${ }^{1)}$
}

\begin{abstract}
Основным результатом статьи можно считать теорему 3, в которой устанавливается справедливость неравенства (2) для полиномов от компонент случайных векторов в пространстве с логарифмически вогнутой плотностью.
\end{abstract}

Ключевые слова и фразы: неравенства типа Хинчина.

Несколько лет тому назад Ю. В. Прохоров исследовал неравенства типа Хинчина.

$$
\left(\mathbf{E}|f(\xi)|^{2}\right)^{1 / 2} \leqslant C(d) \mathbf{E}|f(\xi)|
$$

в классе полиномов $f$ степени $n$. В работе [9], используя разложение по полиномам Эрмита, им установлено такое неравенство в случае нормально распределенных случайных величин $\xi$. В следующей работе [10] с привлечением обобщениых полиномов Лагерра неравенства вида (1) доказаны для случайных величин, имеющих гаммараспределение. Как и собственно результаты, несомненный интерес представляет сам подход к (1), основанный на применении известного неравенства Маркова, связывающего максимальные значения полинома и его производной на конечном интервале. В обоих случаях этот подход позволил получить неравенство (1) с правильным экспоненциальным возрастанием множителей $C(d)$ при растущей степени $d$. Естественным обобщением этих результатов может служить следующее утверждение, доказанное в [2].

Теорема 1. Неравенство (1) справедливо в классе всех полиномов $f$ на $\mathbf{R}$ степени $d$ от произвольньх наборов случайньх величин $\xi=\left(\xi_{1}, \ldots, \xi_{n}\right)$, имекиих $в$ совокупности погарифмически вогнутую плотность ка $\mathbf{R}^{n}$, причем с множителем вида $C(d)=C^{d}$, где $C$ - мекоторая универсальнан постоянная.

Напомним, что неотрицательная функция $\rho$ на $\mathbf{R}^{n}$ называется логарифмически вогнутой, если она удовлетворяет неравенству $\rho((1-t) x+t y) \geqslant \rho(x)^{1-t} \rho(y)^{t}$ при всех $x, y \in \mathbf{R}^{n}$ и $t \in(0,1)$.

В одномерном случае $(n=1)$ теорему 1 можно легко доказать, если не придавать значения скорости роста множителей $C(d)$ (см. [3]). Однако в данном виде утверждение становится намного более деликатным. Впервые вопрос о сравнимости $L^{p}$ - и $L^{1}$-норм - для полиномов от нескольких переменных поо отношению $\mathrm{k}$ равномерному распределению $\lambda_{K}$ на выпуклом компактном теле $K$ в $\mathbf{R}^{n}-6$ бл поставлен В.Д. Мильманом. Решение этой задачи с использованием параметризации Кноте дано Бургеном [4], получившим оценку для вероятностей больших уклонений $\lambda_{K}\{|f| \geqslant t \mathbf{E}|f|\} \leqslant \exp \left\{-t^{c / d}\right\}, t \geqslant t_{0}$, где $c \in(0,1)$ и $t_{0}>0$ - абсолютные постоянные. Эквивалентно, для случайного вектора $\xi$, равномерно распределенного на $K$, справедливо неравенство типа Хинчина

$$
\left(\mathbf{E}|f(\xi)|^{p}\right)^{1 / p} \leqslant C(p, d) \mathbf{E}|f(\xi)|
$$

причем с постоянными вида $C(p, d)=(C p d)^{C d}, C>1$. Emе ранее М.JI. Громов и В.Д. Мильман рассмотрели линейный случай $d=1$ [5]. Из частного случая результата Дж. Бургена (если его применить к равномерному распределению на кубе $[-1,1]^{n}$ при растущей размерности $n$ ) в силу центральной предельной теоремы

* Математический факультет, Сыктывкарский университет, 167001 Сыктывкар, Poссия; Department of Mathematics, Bielefeld University, Bielefeld 33501, Germany.

1) Работа выполнена при поддержке Российского фонда фундаментальных исследований, грант № 99-01-00247. 
вытекает неравенство (1) для нормально распределенных случайных величин $\xi$; однако с худшим ростом констант $C(d)$ по сравнению с результатом Ю. В. Прохорова. Позднее Л. Ловаш и М. Смирновиц [8] ввели так называемый локализационный метод, позволяющий сводить некоторые соотношения между многомерными интегралами $\mathbf{k}$ одномерным (в связи с изопериметрическими задачами на сфере близкие идеи были ранее развиты в [6], см. также [1]). В развитие этого метода Р. Каннан, Л. Ловаш и М. Смирновиц доказали, в частности, одну важную теорему ([7, теорема 2.7]), из которой вытекает следуюшая

Теорема 2. Пусть $p \geqslant 1, C \geqslant 1$, пусть $f$ - кепрерьвкая фужкиия ка $\mathbf{R}^{n}$. Неравенство

$$
\left(\mathbf{E}|f(\xi)|^{p}\right)^{1 / p} \leqslant C \mathbf{E}|f(\xi)|
$$

справедливо для всех случайжых векторов $\xi$, имекоиих логарифмически вогнутую плотность на $\mathbf{R}^{n}$, тогда и только тогда, когда оно справедливо для случайных вехторов вида $\xi=a+\eta b$, где $\eta$ - случайная величияа со значениями в $[0,1]$, имегщая плотность $\lambda e^{-\lambda x} /\left(1-e^{-\lambda}\right), x \in[0,1]\left(a, b \in \mathbf{R}^{n} u \lambda \in \mathbf{R}-\right.$ произвольные параметры).

Если $f=f\left(x_{1}, \ldots, x_{n}\right)$ - полином степени $d$ от $n$ переменных, и $t \in \mathbf{R}$, то $f(a+t b)$ - полином степени не выше $d$ от одного переменного. Поэтому теорема 2 сводит неравенства $(1),(2)$ к размерности $n=1$. Более того, при оценивании постоянных множителей $C(p, d)$, выбирая при необходимости подходящий масштаб, можно предполагать, что случайная величина $\xi$ имеет усеченное показательное распределение $\nu_{n}$ с плотностью

$$
\frac{d \nu_{u}(x)}{d x}=\frac{e^{-x}}{1-e^{-u}} 1_{(0, u)}(x), \quad x \in \mathbf{R}, u>0
$$

(в предельном случае получаем стандартное показательное распределение $\nu_{+\infty}=\boldsymbol{\nu}$ с плотностью $e^{-x}, x>0$ ).

Таким образом, теорема 2 существенно упрощает ситуащию. Однако вопрос об экстремальных полиномах и распределениях, определяющих значение оптимальных констант в (2), все еще остается открытым, и требуется привлечение дополнительных средств при изучении асимптотического поведения $C(p, d)$ как функции двух параметров. Как показано в [2], комбинирование подхода Ю.В. Прохорова с теоремой 2 позволяет получить утверждение теоремы 1 (с постоянной $C=e^{11}$ ). Если применить неравенство (1) последовательно к полиномам $f^{2}, f^{4}, \ldots, f^{2^{k}}$, то при $p \geqslant 2$ мы придем к неравенству (2) с множителями $C(p, d)=p^{C d}$, где $C>1$ - некоторая численная постоянная. В данной заметке мы покажем, как получить более точное утверждение.

Теорема 3. Неравенство (2) справедливо в классе всех полияомов $f$ ка $\mathbf{R}^{n}$ степени $d$ от произволькых случайных векторов $\xi$, имеючих логарифмически вогяутую плотность на $\mathbf{R}^{n}$, причем с множителем вида $C(p, d)=(C p)^{d}$, где $C-$ некоторая универсальяая постоянкая.

Отметим, что, как показывает пример полинома $f(x)=x^{d}$ и случайной величины, имеющей стандартное показательное распределение $\nu$, оптимальная константа в $(2)$ должна удовлетворять неравенству $C(p, d) \geqslant c p^{d} / d^{(p-1) /(2 p)}$ при некоторой $c>0$.

Для доказательства теоремы 3 потребуются некоторые приготовления. Мы опираемся на теоремы 1, 2 и используем рассуждения из [10].

Лемма. Пусть случайяая величия $\xi$ имеет распределение $\nu$. Для яюбого полинома $f$ на $\mathbf{R}$ степени $d \geqslant 1$ и мюбого $p \geqslant 1$

$$
\left(\mathbf{E}|f(\xi)|^{p}\right)^{1 / p} \leqslant\left(e^{12} p\right)^{d} \mathbf{E}|f(\xi)| .
$$

Д ок а з ат ельст в о. Обозначим $\|\eta\|_{p}=\left(\mathbf{E}|\eta|^{p}\right)^{1 / p}$. В силу теоремы 1 , которую мы применяем с $C=e^{11}$, можно предполагать, что $p \geqslant 2$. По однородности 
можно считать, что $\|f(\xi)\|_{2}^{2}=\int_{0}^{+\infty} x^{2}|f(x)|^{2} e^{-x} d x=1$. Введем полиномы Лагерра

$$
L_{k}(x)=\frac{e^{x}}{k !} \frac{d^{k}}{d x^{k}}\left(x^{k} e^{-x}\right)=\sum_{j=0}^{k}(-1)^{j} C_{k}^{j} \frac{x^{j}}{j !}, \quad k=0,1, \ldots
$$

Они образуют полную ортонормальную систему в $L^{2}(\nu)$, причем $f$ допускает представление $f=\sum_{k=0}^{d} a_{k} L_{k}$, где $\sum_{k=0}^{d}\left|a_{k}\right|^{2}=1$. Следовательно, $|f|^{2} \leqslant \sum_{k=0}^{d}\left|L_{k}\right|^{2}$ и

$$
\|f(\xi)\|_{p}^{2}=\left\||f(\xi)|^{2}\right\|_{p / 2} \leqslant \sum_{k=0}^{d}\left\|\left|L_{k}(\xi)\right|^{2}\right\|_{p / 2}=\sum_{k=0}^{d}\left\|L_{k}(\xi)\right\|_{p}^{2}
$$

Из формулы Стирлинга легко вывести, что $\Gamma(p j+1)^{1 / p} \leqslant p^{j} \Gamma(j+1)$ для всех $p \geqslant 2$ и всех целых $j \geqslant 0$. В силу (3) получаем:

$$
\left\|L_{k}(\xi)\right\|_{p} \leqslant \sum_{j=0}^{k} C_{k}^{j} \frac{\left\|\xi^{j}\right\|_{p}}{j !}=\sum_{j=0}^{k} C_{k}^{j} \frac{\Gamma(p j+1)^{1 / p}}{j !} \leqslant \sum_{j=0}^{k} C_{k}^{j} p^{j}=(p+1)^{k} .
$$

Применяя (4), заключаем, что

$$
\|f(\xi)\|_{p}^{2} \leqslant \sum_{k=0}^{d}(p+1)^{2 k} \leqslant(d+1)(p+1)^{2 d}<(2 p)^{2 d}, \quad d \geqslant 2,
$$

и $\|f(\xi)\|_{p}^{2} \leqslant 1+(p+1)^{2}<(2 p)^{2}$ при $d=1$. Таким образом, $\|f(\xi)\|_{p} \leqslant(2 p)^{d}\|f(\xi)\|_{2}$. Остается применить теорему 1 . Лемма доказана.

Д ок а за т е л в с т в о т е о р е м ы 3 . В силу теоремы 2 достаточно установить с некоторой универсальной постоянной $C$ неравенство

$$
\left(\mathbf{E}\left|f\left(\xi_{u}\right)\right|^{p}\right)^{1 / p} \leqslant(C p)^{d} \mathbf{E}\left|f\left(\xi_{u}\right)\right|, \quad p \geqslant 1, u>0
$$

где $f$ - произвольный полином степени $d \geqslant 1$ и $\xi_{u}-$ случайная величина, имеющая распределение $\nu_{u}$. Как и ранее, $\|\eta\|_{p}=\left(\mathbf{E}|\eta|^{p}\right)^{1 / p}(1 \leqslant p \leqslant+\infty)$.

IJae 1: $0<u<24 d$. Пусть $x_{0} \in[0, u]$ - точка максимума $|f|$ на интервале $[0, u]$. Не умаляя обшности $f\left(x_{0}\right)>0$. По неравенству Маркова имеем

$$
\left\|f^{\prime}\left(\xi_{u}\right)\right\|_{\infty}=\max _{0 \leqslant x \leqslant u}\left|f^{\prime}(x)\right| \leqslant \frac{2 d^{2}}{u} \max _{0 \leqslant x \leqslant u}|f(x)|=\frac{2 d^{2}}{u}\left\|f\left(\xi_{u}\right)\right\|_{\infty} .
$$

Следовательно, для всех $x \in[0, u]$

$$
\begin{aligned}
f(x) & \geqslant f\left(x_{0}\right)-\left\|f^{\prime}\left(\xi_{u}\right)\right\|_{\infty}\left|x-x_{0}\right| \\
& \geqslant f\left(x_{0}\right)-\frac{2 d^{2}}{u}\left\|f\left(\xi_{u}\right)\right\|_{\infty}\left|x-x_{0}\right|=\left(1-\frac{2 d^{2}}{u}\left|x-x_{0}\right|\right)\left\|f\left(\xi_{u}\right)\right\|_{\infty} .
\end{aligned}
$$

Поэтому для точек $x$ из интервала $\delta=\left[x_{1}, x_{2}\right] \equiv\left[x_{0}-u /\left(4 d^{2}\right), x_{0}+u /\left(4 d^{2}\right)\right] \cap[0, u]$ верна оценка $f(x) \geqslant \frac{1}{2}\left\|f\left(\xi_{u}\right)\right\|_{\infty}$. Как следствие,

$$
\left\|f\left(\xi_{u}\right)\right\|_{1} \geqslant \int_{\delta} f(x) d \nu_{u}(x) \geqslant \frac{1}{2}\left\|f\left(\xi_{u}\right)\right\|_{\infty} \nu_{u}(\delta) .
$$

Кроме того, так как $x_{2}-x_{1} \geqslant u /\left(4 d^{2}\right)$, для некоторой точки $x_{3} \in\left[x_{1}, x_{2}\right]$ имеем

$$
\nu_{u}(\delta)=\frac{e^{-x_{1}}-e^{-x_{2}}}{1-e^{-u}}=\frac{x_{2}-x_{1}}{1-e^{-u}} e^{-x_{3}} \geqslant \frac{1}{4 d^{2}} \frac{u}{1-e^{-u}} e^{-24 d} \geqslant \frac{1}{4 d^{2}} e^{-24 d} .
$$

Комбинируя с (6), получаем, что $8 d^{2} e^{24 d}\left\|f\left(\xi_{u}\right)\right\|_{1} \geqslant\left\|f\left(\xi_{u}\right)\right\|_{\infty} \geqslant\left\|f\left(\xi_{u}\right)\right\|_{p}$, и (5) легко следует.

Шаг 2: $u \geqslant 24 d$. Пусть $\xi$ - случайная величина, имеющая распределение $\nu$. Применяя неравенство Коши-Буняковского и теорему 1 (снова с $C=e^{11}$ ), имеем

$$
\int_{u}^{\infty}|f(x)| e^{-x} d x \leqslant\|f(\xi)\|_{2}[\nu(u,+\infty)]^{1 / 2} \leqslant e^{11 d-u / 2}\|f(\xi)\|_{1} \leqslant e^{-d}\|f(\xi)\|_{1} .
$$


Поэтому

$$
\frac{1}{1-e^{-u}} \int_{u}^{\infty}|f(x)| e^{-x} d x \leqslant \frac{1}{e\left(1-e^{-24}\right)}\|f(\xi)\|_{1} \leqslant \frac{1}{2}\|f(\xi)\|_{1},
$$

что эквивалентно неравенству $\mathbf{E}\left|f\left(\xi_{u}\right)\right| \geqslant \frac{1}{2} \mathbf{E}|f(\xi)|$. Наконец, в силу леммы

$$
\begin{aligned}
\mathbf{E}\left|f\left(\xi_{u}\right)\right|^{p} & =\frac{1}{1-e^{-u}} \int_{0}^{u}|f(x)|^{p} e^{-x} d x \leqslant \frac{1}{1-e^{-24}} \int_{0}^{\infty}\|f(x)\|^{p} e^{-x} d x \\
& =\frac{1}{1-e^{-24}} \mathbf{E}\|f(\xi)\|^{p} \leqslant 2\left(e^{12} p\right)^{p d}(\mathbf{E}|f(\xi)|)^{p} \leqslant 2^{p+1}\left(e^{12} p\right)^{p d}\left(\mathbf{E}\left|f\left(\xi_{u}\right)\right|\right)^{p} .
\end{aligned}
$$

Приходим к неравенству (5) с $C=4 e^{12}$. Теорема 3 доказана.

Автор хотел бы выразить искреннюю признательность Ю. В. Прохорову за стимулируюшие обсуждения.

\section{СПИСОК ЛИТЕРАТУРЫ}

1. Alesker S. Localization technique on the sphere and the Gromov-Milman theorem on the concentration phenomenon on uniformly convex sphere. - Math. Sci. Res. Inst. Publ., 1999, v. 34, p. 17-27.

2. Bobkov S. G. Remarks on the growth of $L^{p}$-norms of polynomials. - Geometric Aspects of Functional Analysis (Israel Seminar) (to appear).

3. Бобков С. Г., Гётие Ф. О моментах полиномов. - Теория вероятн. и ее примен., 1997, т. 42 , в. 3, с. $638-640$.

4. Bourgain $J$. On the distribution of polynomials on hegh dimensional convex sets. Lecture Notes in Math., 1991, v. 1469, p. 127-137.

5. Gromov M., Milman V.D. Brunn theorem and a concentration of volume phenomena for symmetric convex bodies. - Geometric Aspects of Functional Analysis (Israel Seminar), v. 12, Tel Aviv University, Israel, 1983-1984.

6. Gromov M., Milman V.D. Generalization of the spherical isoperimetric inequality to uniformly convex Banach spaces. - Compositio Math., 1987, v. 62, p. 263-282.

7. Kannan R., Lovász L., Simonovits M. Isoperimetric problems for convex bodies and localization lemma. - Discrete Comput. Geom., 1995, v. 13, p. 541-559.

8. Lovász L., Simonovits $M$. Random walks in a convex body and an improved volume algorithm. - Random Structures Algorithms, 1993, v. 4, № 3, p. 359-412.

9. Прохоров Ю. В. О полиномах от нормально распределенных случайных величин. - Теория вероятн. и ее примен., 1992, т. 37, в. 4, с. 692-694.

10. Прохоров ҒО.В. О полиномах от случайных величин, имеющих гаммараспределение. - Теория вероятн. и ее примен., 1993, т. 38, в. 1, с. 198-202.

Поступила в редакцию 24.V.2000

(C) $2000 \mathrm{r}$.

БОГУСЛАВСКАЯ Е. В.*

\section{ОБ ОПТИМИЗАЦИИ ДОЛГОСРОЧНЫХ НЕОБРАТИМЫХ ИНВЕСТИЩИЙ В ОДНОЙ ДИФФУЗИОННОЙ МОДЕЛИ}

В работе Пиндайка [4] была рассмотрена модель, где главным фактором, вносящим неопределенность, являлся неизвестный заранее требуемый объем инвестиций. В настоящей работе получено точное решение для этой задачи.

* Математический институт им. В. А. Стеклова РАН, ул. Губкина, 8, 117966 Москва, ГСП-1, Россия; e-mail: elenab@mi.ras.ru 